The manuscript was reviewed by a journal (impact factor more than 3.0) but declined for publication. Because it will take some time to address the reviewer comments (requiring experimental work to support theory) and submit again for publication, the manuscript and its supporting information are shared with the community in ChemRxiv. Additional feedback from the community is welcome.

The policy of the journal evaluated the manuscript prevents sharing the reviews. Thus, only a summary of the reviews is provided here, which, the author believes, should be useful for readers. Two reviewers reviewed the manuscript. Both ranked highly on significance. One ranked originality highly and the other ranked originality slightly lower. Major criticisms include presentation needs to improve, and theory should be supported by experimental work. There are some other valuable and easily addressable suggestions. 


\title{
The Ion Pair Thermal Model of MALDI MS
}

Shiyue Fang*

Address reprint requests to:

Shiyue Fang, Department of Chemistry, Michigan Technological University, 1400 Townsend Drive, Houghton, MI 49931 USA

Tel: $906-487-2023$

Email: shifang@mtu.edu

\begin{abstract}
The ion pair thermal model for MALDI MS is described. Key elements of the model include thermal desorption and ionization, strong tendency to neutralization via ion pair formation and proton transfer in the gas phase, thermal equilibrium, overall charge neutral plume, and thermal energy assisted free ion generation via ion pair separation by ion extraction potential. The quantities of ions in the solid sample and in the gaseous plume are estimated. Ion yields of different classes of molecules including peptides, nucleic acids, permanent salts and neutral molecules are estimated at the macroscale and single ion pair levels. The estimated ion yields are close to experimentally observed values under certain assumptions. Explanations of several observations in MALDI MS such as mostly single-charged peaks, improvement of spectra by ammonium cation, and ion suppression are provided. We expect that the model can give insights for the design of new conditions and systems for improving the sensitivity and resolution of MALDI MS and improving its capability and reliability to analyze large biomolecules.
\end{abstract}

\section{Introduction}

MALDI MS has been widely used in the analysis of biomolecules including nucleic acids, peptides, proteins and oligosaccharides. However, after more than 20 years of research, the mechanism of gas phase ion formation in the process of MALDI is still under debate. ${ }^{1-}$ ${ }^{9}$ In most of models proposed in the literature, free ions in the plume were assumed before the application of the ion extraction potential, which implies or gives the impression of an overall charge unbalanced plume. ${ }^{2}$ In this article, the ion pair thermal model is presented. Key assumptions of the model include thermal desorption and ionization, strong tendency to gas phase neutralization, ion pair formation, overall charge balanced plume and thermal equilibrium. Based on the assumptions, attempts are made to provide detailed estimations on the quantities of ions in the gas phase available for extraction and 
detection by the mass spectrometer. The estimations are conducted both at the macroscale and individual ion pair levels. The ion yields derived from the analyses are close to but somewhat lower than experimental data. This may suggest that the preformed ions in the solid sample, the quantities of which are also estimated, ejected into the plume may not completely reach thermal equilibrium in the gas phase and may contribute a portion of the ions that are extracted and detected by the mass spectrometer.

\section{Results and Discussion}

The Ion pair thermal model: Key elements of the ion pair thermal model include: (1) Desorption and ionization are caused by high temperature. (2) The plume is at thermal equilibrium at certain stage of the desorption and ionization process. (3) lons in the plume may come from the solid sample or be formed in gas phase due to thermal equilibrium, and the plume is overall charge neutral. (4) lons in the gas phase have a strong tendency toward neutralization via either proton transfer or ion pair formation. (5) lon to neutral ratio of any species is controlled by gas phase thermal equilibrium. (6) High energy ion pairs are separated by ion extraction potential, and the separated ions are detected by the spectrometer. The following provides more details about the model in the context of information in the literature.

When a MALDI MS sample on a substrate is irradiated with a pulse of laser, photons are absorbed by matrix molecules. The excited matrix molecules relax to ground state rapidly and converts the photo energy to thermal energy, which heats up the sample. According to an estimation based on delivered lase energy, the upper limit of plume temperature could reach as high as $2,700 \mathrm{~K}^{2,1}, 10,11$ The matrix is usually more volatile than the analytes. Rapid evaporation of the matrix molecules brings the less volatile or non-volatile analyte molecules into the gas phase. The thermal desorption may also involve ejection of clusters into the plume. ${ }^{4,12,13}$

It is generally accepted in the literature that direct cation formation by ejection of electrons from matrix molecules is unlikely due to the need of three or more photons for the process to be energetically feasible. For example, the energy of the photon of the commonly used $337 \mathrm{~nm}$ nitrogen laser in MALDI MS is $3.68 \mathrm{eV}$, while the commonly used matrices have an ionization energy of $\sim 8 \mathrm{eV}$ or higher. ${ }^{3,}{ }^{9}$ Various models have been proposed to address the problem. Examples include multiphoton ionization, ${ }^{3,9,14}$ exciton pooling, ${ }^{15,} 16$ excited state proton transfer, ${ }^{3,9}$ ground state autoprotolysis, ${ }^{9}, 17-19$ preformed ion emission, ${ }^{20-22}$ and reduction of charged clusters into individual ions. ${ }^{2,} 4,12,13,23$ Among them, the preformed ion emission model is simple and intuitive. Upon laser irradiation, matrix and analyte ions are ejected into the gas phase along with neutral matrix and analyte molecules. ${ }^{20,22,24}$ The ion pair thermal model considers preformed ions ejected into the gas phase as one of the ion sources.

The ion pair thermal model has a different view about ions in the plume from the previous models. It assumes that individual free ions do not exist in the plume, all ions exist as ion pairs, and the plume is overall charge neutral. lons preformed in the sample can be 
ejected into the gas phase, but they may not contribute to the pool of ions that are extracted and detected by the spectrometer, or they may only contribute a small portion of it, the latter of which is beyond the ion pair thermal model. Once in the gas phase, the preformed ions have a strong tendency to neutralize either through proton transfer or through ion pair formation. Neutral molecules ejected into the gas phase may also be ionized in the plume, and these ions may also neutralize though proton transfer and ion pair formation. Gas phase thermal equilibrium controls ion formation and ion neutralization, and determines the relative amounts of the forms of each species including their ion to neutral ratio. The ion pairs that are not neutralized through proton transfer have different energies. A small portion of the ion pairs that have significantly higher energy than average is separated to free ions by the ion extraction potential, and these free ions are detected by the spectrometer.

The ion pair thermal model does not exclude other pathways of ion formation proposed in the literature,$^{2}$ and overall charged plume may exist and free ions may contribute to the pool of ions that are detected by the spectrometer. For example, when a sample on a metal substrate is sufficiently thin for laser to reach the substrate or part of the substrate is exposed to the laser radiation, electrons, which have a longer free path than other charged species, in the substrate may escape resulting in excess positive charges at the surface of the substrate and in the sample. ${ }^{9,} 25$ Coulombic repulsion leads to desorption resulting an overall positively charged plume. However, in cases that both cations and anions can be detected with similar yields, ${ }^{26}$ the pathway involving overall charged plume may play little roles. The following discussions provide estimations of the types and quantities of ions for several classes of molecules commonly analyzed with MALDI MS. The analyses are conducted for both the solid sample and the gas phase. For the latter, the analysis is based on the assumptions of the ion pair thermal model such as thermal equilibrium. MALDI MS ion yields derived from the analysis are close to but probably somewhat lower than experimentally determined values.

Matrix ionization, ion pair formation, ion neutralization and ion yield estimation: Most matrices used in MALDI MS such as 2,5-dihydroxybenzoic acid (DHB), a-cyano-4hydroxy-cinnamic acid (HCHA), and 3-hydroxypicolinic acid (3-HPA) have a carboxylic acid group. They have the potential to form $[\mathrm{M}-\mathrm{H}]^{-}$(i.e. $\mathrm{RCO}_{2}^{-}$or other forms of deprotonated matrices) and $[\mathrm{M}+\mathrm{H}]^{+}$(i.e. $\mathrm{RCO}_{2} \mathrm{H}_{2}{ }^{+}$or other forms of protonated matrices) via autoprotolysis as shown in equation (1):

$$
[\mathrm{M}-\mathrm{H}]^{-}+[\mathrm{M}+\mathrm{H}]^{+} \rightleftharpoons[\mathrm{M}-\mathrm{H}]^{-}:[\mathrm{M}+\mathrm{H}]^{+} \rightleftharpoons \mathrm{M}+\mathrm{M}
$$

Where $\mathrm{M}$ is a matrix molecule. $[\mathrm{M}-\mathrm{H}]^{-}:[\mathrm{M}+\mathrm{H}]^{+}$is the ion pair of ions $[\mathrm{M}-\mathrm{H}]^{-}$and $[\mathrm{M}+\mathrm{H}]^{+}$. Before laser irradiation, due to ion stabilization by residue solvents, polar neutral matrix molecules and polar neutral analyte molecules, in solid phase a fraction of the matrices exists in the forms of $[\mathrm{M}-\mathrm{H}]^{-}$and $[\mathrm{M}+\mathrm{H}]^{+}$. According to the preformed ion emission model, ${ }^{20,22,24}$ these ions will enter the gas phase upon laser irradiation. Once in the gas phase, the shielding molecules will fall off due to the high temperature, which was estimated to be as high as $2,200 \mathrm{~K}$ at the early stage of the plume, ${ }^{2}$ and the weakness of 
the attracting forces between the neutral shielding molecules and the ions. Therefore, there is a strong tendency for the ions $[\mathrm{M}-\mathrm{H}]^{-}$and $[\mathrm{M}+\mathrm{H}]^{+}$to form the ion pair $[\mathrm{M}-\mathrm{H}]:[\mathrm{M}+\mathrm{H}]^{+}$ and go back to $\mathrm{M}$ via proton transfer. Both these processes neutralize the ions.

The amount of $[\mathrm{M}-\mathrm{H}]^{-}$and $[\mathrm{M}+\mathrm{H}]^{+}$in the solid sample is not easy to determine, ${ }^{24}$ but can be roughly estimated using the equilibrium constant of equation (1), which can be estimated using the $p K_{a}$ of the matrices. For example, the $p K_{a}$ of CHCA in water is $1.17,{ }^{27}$ which corresponds to an equilibrium constant of $6.8 \times 10^{-2}$ and a concentration of $0.26 \mathrm{M}$ for $[\mathrm{M}-\mathrm{H}]^{-}$and $[\mathrm{M}+\mathrm{H}]^{+}$with the assumption that the $\mathrm{p} K_{\mathrm{a}}$ value in water can be used here even though the proton goes to the matrix molecules (all calculations in the article are given in supporting information). The concentration corresponding to ion to neutral ratio of $3.5 \times 10^{-2}$ using $133 \mathrm{~mL} / \mathrm{mol}$ as the molar volume of CHCA. Evidently, the actual concentration of the ions and thus the ion to neutral ratio should be significantly lower because the values of $\mathrm{p} K_{\mathrm{a}}$ of carboxylic acids in acids are usually two or more units larger than those in water. ${ }^{28}$ In addition, the $\mathrm{pK}$ auto of acetic acid has been suggested to be 14.5. ${ }^{29}$ Assuming the $\mathrm{pK}$ auto of $\mathrm{CHCA}$ is close to that number, the concentration of $[\mathrm{M}-\mathrm{H}]^{-}$ and $[\mathrm{M}+\mathrm{H}]^{+}$would be in the order of $10^{-7}$. However, it has been suggested that small amount of residue solvents especially water can drastically change $p K_{a}$ values of acids. ${ }^{29}$ MALDI samples are usually prepared from solutions that contain water, and the samples are usually not dried extensively before MALDI MS experiments. It is reasonable to assume that the crystals contain significant amount of water. Thus, the ion concentration should be significantly higher than $10^{-7}$.

The tendency for ion neutralization via ion pair formation in the gas phase can be estimated using the equilibrium constant between the ion pair $[\mathrm{M}-\mathrm{H}]^{-}:[\mathrm{M}+\mathrm{H}]^{+}$and the ions $[\mathrm{M}-\mathrm{H}]^{-}$and $[\mathrm{M}+\mathrm{H}]^{+}$in equation (1). Assuming the distance between the ions in an ion pair is $1 \mathrm{~nm}$ and the plume temperature is $1,000 \mathrm{~K}$, the energy needed to separate the ion pair into free ions is $138 \mathrm{~kJ} / \mathrm{mol}$, which corresponds to an equilibrium constant of $10^{7}$ favoring the neutral ion pair. If a distance of $0.5 \mathrm{~nm}$ is assumed, the numbers become $276 \mathrm{~kJ} / \mathrm{mol}$ and $10^{14}$, respectively (calculations are given in supporting information).

The tendency for ion neutralization via proton transfer in the gas phase can be estimated using the equilibrium constant between the neutral matrix molecule $M$, and the ions [M$\mathrm{H}]^{-}$and $[\mathrm{M}+\mathrm{H}]^{+}$in equation (1). In gas phase, $\mathrm{pK}$ a values are given in Gibbs energy, and those for carboxylic acids are around $1350 \mathrm{~kJ} / \mathrm{mol}$ (see supporting information). ${ }^{30,} 31$ Proton affinity of double bonded oxygen is around $719 \mathrm{~kJ} / \mathrm{mol} .{ }^{32}$ Thus, the equilibrium constant is $-631 \mathrm{~kJ} / \mathrm{mol}$ or $10^{33}$ assuming that the plume temperature is $1,000 \mathrm{~K}$. At 2,200 $\mathrm{K}$, the numbers are is $-640 \mathrm{~kJ} / \mathrm{mol}$ and $10^{16}$, respectively. In both cases, the equilibrium strongly favors the neutral matrix molecules.

Assuming thermal equilibrium is fully achieved at certain stage between laser irradiation and ion extraction in the gas phase, the estimated ion yield at $1,000 \mathrm{~K}$ is $10^{-16}$ (see supporting information for calculation), which is much lower than the experimentally determined ion yields of $10^{-7}-10^{-8} .{ }^{23}$ At $2,200 \mathrm{~K}$, the estimated ion yield is $10^{-8}$, which is consistent with the experimental number. However, the actual temperature may be lower, 
and if that is the case, a portion of the ions detected may be from the solid sample, in which the ion to neutral ratio is higher due to shielding by neutral molecules.

Peptide ionization, ion pair formation, ion neutralization and ion yield estimation: Peptides contain ionizable acidic and basic groups such as carboxylic acid and amine groups. For carboxylic acid groups, they exist in protonated and deprotonated forms as shown in equations (2-4):

$$
\begin{aligned}
& \text { Pep }-\mathrm{CO}_{2}^{-}+\mathrm{Met}^{+} \rightleftharpoons \text { Pep-CO}{ }^{-}{ }^{-} \text {Met }^{+} \\
& \text {Pep- }-\mathrm{CO}_{2}^{-}+\mathrm{R}_{3} \mathrm{NH}^{+} \rightleftharpoons \mathrm{Pep}-\mathrm{CO}_{2}^{-}{ }^{-} \mathrm{R}_{3} \mathrm{NH}^{+} \\
& \rightleftharpoons \mathrm{Pep}-\mathrm{CO}_{2} \mathrm{H}+\mathrm{R}_{3} \mathrm{~N} \\
& \text { Pep- } \mathrm{CO}_{2}{ }^{-}+[\mathrm{M}+\mathrm{H}]^{+} \rightleftharpoons \text { Pep- }-\mathrm{CO}_{2}^{-}{ }^{-} \mathrm{i}[\mathrm{M}+\mathrm{H}]^{+} \\
& \rightleftharpoons \text { Pep }-\mathrm{CO}_{2} \mathrm{H}+\mathrm{M}
\end{aligned}
$$

Where Pep stands for peptide. Met is metal cations such as $\mathrm{Na}^{+}$and $\mathrm{K}^{+}$. $\mathrm{R}_{3} \mathrm{NH}^{+}$is nitrogen associated cations such as ammonium, guanidium, pyridinium and imidazolium ions. Pep- $\mathrm{CO}_{2}^{-}: \mathrm{Met}^{+}, \mathrm{Pep}-\mathrm{CO}_{2}^{-}: \mathrm{R}_{3} \mathrm{NH}^{+}$, and $\mathrm{Pep}-\mathrm{CO}_{2}^{-}:[\mathrm{M}+\mathrm{H}]^{+}$are ion pairs. In solid phase before laser irradiation, due to solvation by residue solvents and shielding by neutral polar matrix and analyte molecules, even in the medium of the usually acidic matrices, there is certain amount of well isolated ions. Upon laser irradiation, once the analyte goes into the gas phase, residue solvents and neutral shielding molecules fall off, and the ions form tightly associated ion pairs. In the case of $\mathrm{Met}^{+}$, the ion pair remains intact (equation 2). In the cases of $\mathrm{R}_{3} \mathrm{NH}^{+}$and $[\mathrm{M}+\mathrm{H}]^{+}$, the ion pairs may convert to neutral molecules via proton transfer (equations 3-4).

The amount of Pep- $\mathrm{CO}_{2}{ }^{-}$in the solid sample can be estimated using the $\mathrm{pK}$ a of carboxylic acid group in amino acids. Assuming that the $\mathrm{pK}$ a of $\mathrm{Pep}_{-} \mathrm{CO}_{2}{ }^{-}$is 2.2 and the concentration of $[\mathrm{M}+\mathrm{H}]^{+}$in the sample with $\mathrm{CHCA}$ being the matrix is $0.26 \mathrm{M}$ as discussed earlier, the concentration of Pep- $\mathrm{CO}_{2}^{-}$would be $7.3 \times 10^{-5} \mathrm{M}$ when $10 \mathrm{pmol}$ of peptide with one carboxylic acid group is dispersed in $25 \mathrm{nmol}$ of $\mathrm{CHCA}^{23}$ and $133 \mathrm{~mL} / \mathrm{mole}$ is taken as the molar volume of $\mathrm{CHCA}$. It is noted that the concentration of $\mathrm{Pep}-\mathrm{CO}_{2}-$ may be far away from $7.3 \times 10^{-5} \mathrm{M}$ because the sample is a solid and it contains other materials besides the peptide and the matrix.

The tendency for ion neutralization in gas phase via the formation of the ion pairs in equations (2-4) should be similar as discussed for matrix ionization, and the equilibrium constant should be in the order of $10^{-7}$ disfavoring the free ions. The tendency for ion neutralization in the gas phase via proton transfer as described in equations (3-4) can be estimated by the corresponding equilibrium constants, and they are $-400 \mathrm{~kJ} / \mathrm{mol}$ or $10^{21}$ and $-584 \mathrm{~kJ} / \mathrm{mol}$ or $10^{30}$, respectively when $\mathrm{p} K_{\mathrm{a}}$ of $903 \mathrm{~kJ} / \mathrm{mol}$ of guanidinium is used for $\mathrm{R}_{3} \mathrm{NH}^{+},{ }^{33} \mathrm{pK}$ a of $1303 \mathrm{~kJ} / \mathrm{mol}$ is used for Pep-COOH, ${ }^{31} \mathrm{pK}$ a of $719 \mathrm{~kJ} / \mathrm{mol}$ is used for $[\mathrm{M}+\mathrm{H}]^{+}, 32$ and the plume temperature is assumed to be $1,000 \mathrm{~K}$. Under the same 
assumptions but with the temperature being $2,200 \mathrm{~K}$, the numbers become $-462 \mathrm{~kJ} / \mathrm{mol}$ or $10^{11}$ for equation (3), and $-593 \mathrm{~kJ} / \mathrm{mol}$ or $10^{14}$. In all cases, the equilibria strongly favor the neutral species.

Assuming thermal equilibrium is fully achieved, at $1,000 \mathrm{~K}$, the ion yields estimated according to equations (3) and (4) are $10^{-10}$ and $10^{-14}$, respectively. These numbers are significantly lower than experimental values $\left(\sim 10^{-4}\right) \cdot{ }^{34}$ At $2,200 \mathrm{~K}$, the estimated ion yields are $10^{-5}$ and $10^{-6}$ according to equations (3) and (4), respectively. These numbers are close to experimental values. As discussed earlier, the plume temperature may be lower than $2,200 \mathrm{~K}$, and thus, the estimated ion yields may be lower than the observed numbers. Therefore, thermal equilibrium may not be fully achieved in the plume, and a portion of the ions detected may be from the sample. In addition, equation (2), where neutralization via proton transfer is impossible, may be responsible for the lower than observed ion yield.

For amine, imidazole and guanidine groups, they may exist in protonated and unprotonated forms as shown in equations (5-6):

$$
\begin{aligned}
& \text { Pep-NH }{ }^{+}+\mathrm{X}^{-} \rightleftharpoons \text { Pep- } \mathrm{NH}^{+}: \mathrm{X}^{-} \rightleftharpoons \text { Pep-N + HX } \\
& \text { Pep-NH+ }{ }^{+}+[\mathrm{M}-\mathrm{H}]^{-} \rightleftharpoons \text { Pep-NH }{ }^{+}:[\mathrm{M}-\mathrm{H}]^{-} \\
& \rightleftharpoons \text { Pep-N + M }
\end{aligned}
$$

Where Pep- $\mathrm{NH}^{+}$and Pep-N stands for protonated and unprotonated amine, imidazole and guanidine groups in peptides. $\mathrm{X}^{-}$stands for anions such as halides, phosphate, hydrogen phosphates, sulfate, hydrogen sulfate, nitrate, hydroxide, and carboxylates. In solid phase with the usually acidic matrix as the media, Pep-N is mostly protonated, and the isolated ions predominates. Upon laser irradiation, in the gas phase the equilibria represented by equations (5-6) will have a strong tendency toward ion pairs and neutral molecules.

The amount of Pep- $\mathrm{NH}^{+}$in the solid sample should be close to $100 \%$ because the $\mathrm{pK}$ a of the matrix such as CHCA is about 1.17 as described earlier, ${ }^{27}$ and that of $\mathrm{Pep}-\mathrm{NH}^{+}$can be as high as 14. Again, these numbers are for the cases with water as the medium and thus not accurate for solid CHCA sample. However, it should be reasonable to assume that a high percentage of peptide analytes exists in the form of $\mathrm{Pep}-\mathrm{NH}^{+}$in the sample in the context that the MALDI MS ion yields of peptides are in the order of $10^{-4} .{ }^{34}$

In the gas phase, the tendency for neutralization via ion pair formation in equations (5-6) should be similar as discussed earlier. The tendency for neutralization via proton transfer as shown in equations (5-6) can be estimated using the corresponding equilibrium constants, and they are $-291 \mathrm{~kJ} / \mathrm{mol}$ or $10^{15}$ and $-447 \mathrm{~kJ} / \mathrm{mol}$ or $10^{23}$, respectively when a $\mathrm{pK}$ a of $903 \mathrm{~kJ} / \mathrm{mol}$ of guanidinium is used for Pep- $\mathrm{NH}^{+}, 33 \mathrm{pK}$ of $1194 \mathrm{~kJ} / \mathrm{mol}$ of sulfuric acid is used for $\mathrm{HX},{ }^{35} \mathrm{pK}$ a of $1350 \mathrm{~kJ} / \mathrm{mol}$ of carboxylic acid is used for $\mathrm{M},{ }^{31}$ and the plume temperature is assumed to be $1,000 \mathrm{~K}$. At $2,200 \mathrm{~K}$, the numbers become $-347 \mathrm{~kJ} / \mathrm{mol}$ or 
$10^{8}$ for the case of equation (5) and $-509 \mathrm{~kJ} / \mathrm{mol}$ or $10^{12}$ for the case of equation (6). In both cases, the equilibria strongly favor the neutral species.

Assuming thermal equilibrium is fully achieved, at $1,000 \mathrm{~K}$, the ion yields estimated according to equations (5) and (6) are $10^{-8}$ and $10^{-7}$, respectively. These numbers are significantly lower than experimental values $\left(\sim 10^{-4}\right) \cdot{ }^{34}$ At $2,200 \mathrm{~K}$, the estimated ion yields are $10^{-4}$ in both cases. The number is consistent with experimental values. As discussed earlier, the plume temperature may be lower than $2,200 \mathrm{~K}$, and thus, the estimated ion yields may be lower than the observed numbers and ions directly from the solid sample may be needed to justify a portion of the observed ion yields.

Nucleic acid ionization, ion pair formation, ion neutralization and ion yield estimation: Nucleic acids contain easily ionizable phosphate groups. In addition, ionization can also occur at the exo-amino groups of adenine, guanine and cytoside, at the $\mathrm{N}-7$ position of purines via protonation, and at other electronegative locations. For phosphate groups, they may exist in protonated and deprotonated forms as shown in equations (7-9):

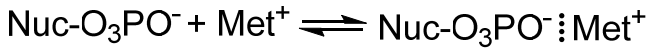

$$
\begin{aligned}
& \text { Nuc- }{ }_{3} \mathrm{PO}^{-}+\mathrm{R}_{3} \mathrm{NH}^{+} \rightleftharpoons \mathrm{Nuc}-\mathrm{O}_{3} \mathrm{PO}^{-} \vdots \mathrm{R}_{3} \mathrm{NH}^{+} \\
& \rightleftharpoons \mathrm{Nuc}-\mathrm{O}_{3} \mathrm{POH}+\mathrm{R}_{3} \mathrm{~N} \\
& \mathrm{Nuc}-\mathrm{O}_{3} \mathrm{PO}^{-}+[\mathrm{M}+\mathrm{H}]^{+} \rightleftharpoons \mathrm{Nuc}-\mathrm{O}_{3} \mathrm{PO}^{-}:[\mathrm{M}+\mathrm{H}]^{+} \\
& \rightleftharpoons \mathrm{Nuc}-\mathrm{O}_{3} \mathrm{POH}+\mathrm{M}
\end{aligned}
$$

Where Nuc stands for nucleic acid (DNA and RNA). In solid phase with the usually acidic matrix as the media, some of the phosphate groups exist in deprotonated form. The counter cations can be $\mathrm{Met}^{+}, \mathrm{R}_{3} \mathrm{NH}^{+}$and $[\mathrm{M}+\mathrm{H}]^{+}$, which are defined earlier. Upon laser irradiation, in the gas phase the equilibrium represented by equations (7-9) will have a strong tendency toward ion pairs. In the case of equation (7), the ion pair remains. In the cases of equations (8-9), the ion pairs can further convert to neutral molecules via proton transfer.

The concentration of Nuc- $\mathrm{O}_{3} \mathrm{PO}^{-}$in the solid sample can be estimated using the $\mathrm{pKa}$ of

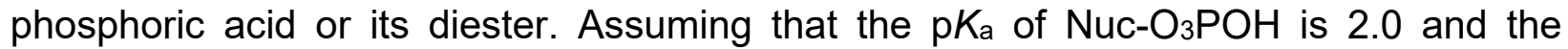
concentration of $[\mathrm{M}+\mathrm{H}]^{+}$in the sample with 3-HPA being the matrix is $0.27 \mathrm{M}$ (see supporting information), the concentration of $\mathrm{Nuc}_{-} \mathrm{O}_{3} \mathrm{PO}^{-}$would be $1.6 \times 10^{-4} \mathrm{M}$ when the amount of nucleic acid containing $10 \mathrm{pmol}$ of phosphate diester group is dispersed in 25 nmol of 3-HPA, $93.6 \mathrm{~mL} / \mathrm{mole}$ is taken as the molar volume of 3 -HPA, and 1.14 is assumed as the $\mathrm{pK}_{\mathrm{a}}$ of 3 -HPA. It is noted that the concentration of Nuc- $\mathrm{O}_{3} \mathrm{PO}^{-}$may be far away from $1.6 \times 10^{-4} \mathrm{M}$ because the sample is a solid and it contains other materials besides the nucleic acid and the matrix. 
In the gas phase, the tendency for neutralization via ion pair formation in equations (7-9) should be similar as discussed earlier. The tendency for neutralization via proton transfer as shown in equations (8-9) can be estimated by the corresponding equilibrium constants, and they are $-557 \mathrm{~kJ} / \mathrm{mol}$ or $10^{29}$ and $-577 \mathrm{~kJ} / \mathrm{mol}$ or $10^{30}$, respectively when a $\mathrm{pKa}$ of

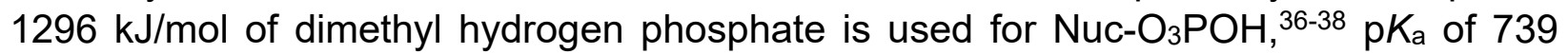
$\mathrm{kJ} / \mathrm{mol}$ of ammonium is used for $\mathrm{R}_{3} \mathrm{NH}^{+}, 33,39,40 \mathrm{pK}$ a of $719 \mathrm{~kJ} / \mathrm{mol}$ of carboxylic acid is used for $[\mathrm{M}+\mathrm{H}]^{+}, 32$ and the plume temperature is assumed to be $1,000 \mathrm{~K}$. At $2,200 \mathrm{~K}$, under otherwise the same conditions, the numbers become $-585 \mathrm{~kJ} / \mathrm{mol}$ or $10^{14}$ for the case of equation (8) and $-593 \mathrm{~kJ} / \mathrm{mol}$ or $10^{14}$ for the case of equation (9). In all cases, the equilibria strongly favor the neutral species.

Assuming thermal equilibrium is fully achieved, at $1,000 \mathrm{~K}$, the ion yields estimated according to equations (8) and (9) are both in the order of $10^{-14}$. At 2,200 K, the estimated ion yields are $10^{-7}$ and $10^{-6}$, respectively. Ion yields for nucleic acids have not been determined using experimental methods.

For the basic nitrogen atoms in the nucleobases, they can exist in protonated and unprotonated forms as shown in equations (10-11):

$$
\begin{aligned}
& \text { Nuc-NH }{ }^{+}+X^{-} \rightleftharpoons \text { Nuc-NH } H^{+} ! X^{-} \\
& \rightleftharpoons \text { Nuc- } \mathrm{N}+\mathrm{HX}
\end{aligned}
$$

Nuc-NH ${ }^{+}+[\mathrm{M}-\mathrm{H}]^{-} \rightleftharpoons \mathrm{Nuc}-\mathrm{NH}^{+} ![\mathrm{M}-\mathrm{H}]^{-}$

$\rightleftharpoons \mathrm{Nuc}-\mathrm{N}+\mathrm{M}$

Where $X^{-}$are the same as defined earlier, and include phosphate groups in the nucleic acid analyte. In the solid sample, some of the nitrogen atoms in the analyte are protonated, and there should be substantial number of nucleobases that carry a positive charge. The charged moiety of the nucleobases and their counter anions may be well separated. Upon laser irradiation, in the gas phase, tight ion pairs form, which can further convert to neutral species via proton transfer (equations 10-11).

The amount of Nuc- $\mathrm{NH}^{+}$in the solid sample should be high because the $\mathrm{p} K_{\mathrm{a}}$ of the matrix

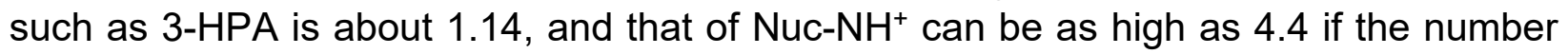
for cytosine is used..$^{41}$ Again, these numbers are for the cases with water as the medium and thus not accurate for solid MALDI samples. In addition, nucleic acid samples usually contain other materials such as residue solvents and co-matrices, which further make estimation difficult.

In the gas phase, the tendency for neutralization via ion pair formation in equations (1011) should be similar as discussed earlier. The tendency for neutralization via proton transfer as shown in equations (10-11) can be estimated using the corresponding equilibrium constants, and they are $-334 \mathrm{~kJ} / \mathrm{mol}$ or $10^{17}$ and $-490 \mathrm{~kJ} / \mathrm{mol}$ or $10^{26}$, respectively when a $\mathrm{pK} a$ of $860 \mathrm{~kJ} / \mathrm{mol}$ for the protonated N-7 of guanine, ${ }^{42}$ is used for Nuc- $\mathrm{NH}^{+}, \mathrm{pK} a$ of $1194 \mathrm{~kJ} / \mathrm{mol}$ of sulfuric acid is used for $\mathrm{HX},{ }^{35} \mathrm{pK} a$ of $1350 \mathrm{~kJ} / \mathrm{mol}$ of 
carboxylic acid is used for $\mathrm{M},{ }^{31}$ and the plume temperature is assumed to be $1,000 \mathrm{~K}$. At $2,200 \mathrm{~K}$, the numbers become $-329 \mathrm{~kJ} / \mathrm{mol}$ or $10^{8}$ for the case of equation (10), and -490 $\mathrm{kJ} / \mathrm{mol}$ or $10^{12}$ for the case of equation (11). In all the cases, the equilibria strongly favor the neutral species.

Assuming thermal equilibrium is fully achieved, at $1,000 \mathrm{~K}$, the ion yields estimated according to equations (10) and (11) are $10^{-8}$ and $10^{-10}$, respectively. At $2,200 \mathrm{~K}$, the estimated ion yields both become $10^{-4}$.

Neutral molecule ionization, ion pair formation, ion neutralization and ion yield estimation: For analytes that lack acidic groups such as carboxylic, phosphoric and sulfuric acid groups, contribution of ions from autoprotolysis will be limited. In the cases that the analytes have electron donor groups such as ether, alcohol, aldehyde, ketone, ester, amine, amide, nitro, cyano, guanidine, imidazole and pyridine groups, protonation by acidic matrix molecules and coordination with metal cations from residue salts can be the major pathway for ionization (equations 12-14).

$$
\begin{aligned}
& \mathrm{AH}^{+}+[\mathrm{M}-\mathrm{H}]^{-} \rightleftharpoons \mathrm{A} \mathrm{H}^{+} \vdots[\mathrm{M}-\mathrm{H}]^{-} \\
& \rightleftharpoons \mathrm{A}+\mathrm{M} \\
& \mathrm{AH}^{+}+\mathrm{X}^{-} \rightleftharpoons \mathrm{AH} \mathrm{AH}^{+} \vdots \mathrm{X}^{-} \\
& \rightleftharpoons \mathrm{A}+\mathrm{HX} \\
& \mathrm{A}+\mathrm{Met}^{+}+\mathrm{X}^{-} \rightleftharpoons \mathrm{A}{\mathrm{M} \mathrm{Met}^{+}+\mathrm{X}^{-}}^{\rightleftharpoons} \mathrm{A}_{\mathrm{Met}}^{+} \vdots \mathrm{X}^{-}
\end{aligned}
$$

Where A stands for analyte, and $M$ stands for a matrix that contains an acidic group such as carboxylic acid group. Met $^{+}$and $\mathrm{X}^{-}$are as defined earlier. In the solid sample, the ratio of isolated $\mathrm{AH}^{+},[\mathrm{M}-\mathrm{H}]^{-}, \mathrm{Met}^{+}, \mathrm{X}^{-}$and $\mathrm{A}^{\prime} \mathrm{Met}^{+}$surrounded and stabilized by neutral matrix molecules over neutral species can be low but can be considered significant when compared with the ion yield detectable by MS, which can be as low as $10^{-8}$. Upon laser irradiation, in the gas phase, $\mathrm{AH}^{+}$and $[\mathrm{M}-\mathrm{H}]^{-}$, and $\mathrm{AH}^{+}$and $\mathrm{X}^{-}$will become more associated with each other to form ion pairs, which will have a strong tendency to form the neutral molecules via proton transfer (equations 12-13). The ions and ionic species $\mathrm{Met}^{+}, \mathrm{X}^{-}$and $A \vdots$ Met $^{+}$will have a strong tendency to form the neutral $A \vdots$ Met $^{+}: X^{-}$, which is more stable than isolated $A, \mathrm{Met}^{+}$, and $X^{-}$, or as isolated $A^{\prime} \mathrm{Met}^{+}$and $\mathrm{X}^{-}$(equation 14). Polyethylene glycol (PEG), oligosaccharides, and polystyrene belong to this group of analytes. Polystyrene lacks electron pair for proton and main group metal cations, but can coordinate with transition metals using its $\pi$ and $\pi^{*}$ orbitals.

The amount of $\mathrm{AH}^{+}$and $\mathrm{A}: \mathrm{Met}^{+}$in the solid sample can be estimated using the corresponding equilibrium constants of equations (12-14). For $\mathrm{AH}^{+}$, in an exemplary case of $\mathrm{A}$ being diethyl ether, which is one of the weakest coordinating molecules, and $\mathrm{M}$ being $\mathrm{CHCA}$, the concentration of $\mathrm{AH}^{+}$would be $2.4 \times 10^{-4} \mathrm{M}$ assuming that the $\mathrm{pK}$ a of -3.5 in water for the conjugate acid of diethyl ether ${ }^{43,44}$ and 1.17 in water for $\mathrm{CHCA}^{27}$ can be 
used here, and the sample consists 10 pmol analyte in $25 \mathrm{nmol}$ matrix. For $\mathrm{A}: \mathrm{Met}^{+}$, in an exemplary case of $A$ being $15-$ crown-5, $\mathrm{Met}^{+}$being $\mathrm{Na}^{+}$, and $\mathrm{M}$ being $\mathrm{CHCA}$, the concentration of $A: \mathrm{Met}^{+}$would be $2.2 \times 10^{-5} \mathrm{M}$, which corresponds to a ratio of $7.3 \times 10^{-3}$

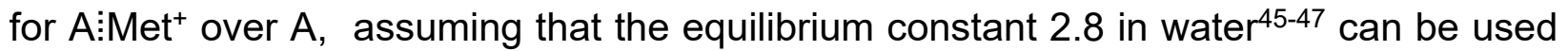
for equation (14), $1 \mu \mathrm{L}$ typical LC-MS grade water with $0.2 \mathrm{ppm} \mathrm{Na}{ }^{+}$is used to prepare the sample, and the sample consists $10 \mathrm{pmol}$ analyte in $25 \mathrm{nmol} \mathrm{CHCA}$ (molar volume $133 \mathrm{~mL} / \mathrm{mol}$ ). Again, it is noted that the $\mathrm{p} K_{\mathrm{a}}$ values and equilibrium constant from the literature may be significantly different from the actual values in solid MALDI MS samples and estimations may be far away from being accurate.

In the gas phase, for the case of equation (12), we can use the example of $A$ being diethyl ether and $\mathrm{M}$ being $\mathrm{CHCA}$ to illustrate the tendency toward neutralization via proton transfer. The equilibrium constant is $-613 \mathrm{~kJ} / \mathrm{mol}$ or $10^{32}$ when the $\mathrm{p} K_{\mathrm{a}}$ of $737 \mathrm{~kJ} / \mathrm{mol}$ for protonated diethyl ether ${ }^{33,48-50}$ is used for $\mathrm{AH}^{+}, \mathrm{pK}$ a of $1350 \mathrm{~kJ} / \mathrm{mol}$ of carboxylic acid is used for $\mathrm{M},{ }^{31}$ and the plume temperature is assumed to be $1,000 \mathrm{~K}$. For the case of equation (13), under the same assumptions for equation (12) but with the counter anion being $X^{-}$and with $X^{-}$being hydrogen sulfate, the equilibrium constant is $-457 \mathrm{~kJ} / \mathrm{mol}$ or $10^{24}$. For the case of equation (14), we can use the example of $A$ being $15-c r o w n-5$ and $\mathrm{Met}^{+}$being $\mathrm{Na}^{+}$. The identity of $\mathrm{X}^{-}$is insignificant here. The equilibrium constant for the formation of $A: \mathrm{Met}^{+}$is $-159 \mathrm{~kJ} / \mathrm{mol}^{51}$ or $10^{8}$ favoring the $A: \mathrm{Met}^{+}$assuming the plume temperature is $1,000 \mathrm{~K}$. In this case, neutralization via proton transfer in the gas phase cannot occur. Neutralization via ion pair formation to give $A:$ Met $^{+}: \mathrm{X}^{-}$is similar as discussed earlier. At $2,200 \mathrm{~K}$, the numbers become $-606 \mathrm{~kJ} / \mathrm{mol}$ or $10^{14}$ for the case of equation (12), and $-445 \mathrm{~kJ} / \mathrm{mol}$ or $10^{10}$ for the case of equation (13).

Assuming thermal equilibrium is fully achieved, at $1,000 \mathrm{~K}$, the ion yields estimated according to equations (12) and (13) are $10^{-16}$ and $10^{-12}$, respectively. At $2,200 \mathrm{~K}$, the estimated ion yields become $10^{-6}$ for the case of equation (12) and $10^{-5}$ for the case of equation (13). For the case of equation (14), after making a number of assumptions such as the amount of sodium chloride in the sample, the plume pressure at the time thermal equilibrium is achieved and others, the ion yield is estimated to be $2.9 \times 10^{-5}$ (see supporting information for calculations).

Permanent salt ionization, ion pair formation, ion neutralization and ion yield estimation: For permanent salts such as benzyltriphenylphosphonium chloride (BTPP$\mathrm{Cl}$, 1-butyl-3-methylimidazolium hexafluorophosphate and trihexyltetradecylphosphonium bis(2,4,4-trimethylpentyl)phosphinate, the behavior of their ions in a matrix, which has an acidic proton, can be described with equations (1517):

$$
\begin{aligned}
& \mathrm{A}^{+}+\mathrm{A}^{-} \rightleftharpoons \mathrm{A}^{+} \vdots \mathrm{A}^{-} \\
& \mathrm{A}^{+}+[\mathrm{M}-\mathrm{H}]^{-} \rightleftharpoons \mathrm{A}^{+} \vdots[\mathrm{M}-\mathrm{H}]^{-} \\
& \mathrm{A}^{-}+[\mathrm{M}+\mathrm{H}]^{+} \rightleftharpoons \mathrm{A}^{-} \vdots[\mathrm{M}+\mathrm{H}]^{+}
\end{aligned}
$$


Where $\mathrm{A}^{+}$stands for analyte cations such as BTPP+ $\mathrm{A}^{-}$is analyte anions such as $\mathrm{Cl}^{-}$. $A^{+} \vdots A^{-}, A^{+}:[M-H]^{-}, A^{-}:[M+H]^{-}$are ion pairs. In solid phase, isolated ions predominate due to stabilization by the neutral polar matrix molecules. They are close to $100 \%$. Upon laser irradiation, the ions will form ion pairs, which are regarded as neutral species. The equilibrium constants for the ion pair formation are similar to the cases discussed earlier.

Assuming thermal equilibrium is fully achieved in the plume, the interion distance in the ion pair is $1 \mathrm{~nm}$, the temperature is $1,000 \mathrm{~K}$, the sample composition is $10 \mathrm{pmol}$ analyte in $25 \mathrm{nmol}$ matrix, and plume pressure at the time thermal equilibrium is achieved and ratios of species in the plume are maintained until ion extraction is $10 \mathrm{~atm}$, the estimated ion yield is $5.1 \times 10^{-3}$, which is close to but somewhat higher than the experimentally determined value $10^{-4}{ }^{23}$ If $0.5 \mathrm{~nm}$ is taken as the interion distance and the pressure is 10 atm, the estimated ion yield is $1.6 \times 10^{-6}$.

Dependence of gas phase equilibrium on temperature: For all the above ionization situations, a common feature is that the plume strongly favors neutral species, which is achieved by proton transfer or by ion pair formation. However, because all the neutralization processes are exothermic, if temperature increases, the neutralization processes become less favored. For example, the equilibrium constant of ion pair formation (e.g. the one in equation 1) may drop from $10^{7}$, the value of $1,000 \mathrm{~K}$, to $10^{4}$ when the temperature is $1,800 \mathrm{~K}$. The equilibrium constant of equation (5) may drop from $10^{15}$, the value of $1,000 \mathrm{~K}$, to $10^{9}$ when the temperature is $1,800 \mathrm{~K}$ (see supporting information). If the partial pressures of the two species on either side of equation (5) were assumed identical, the ratio of Pep- $\mathrm{NH}^{+}$over Pep-N would be $10^{-5}$, which is close to the experimentally determined ion yields of peptides in MALDI MS. ${ }^{34}$ Therefore, the reduced tendency of neutralization under higher temperature is beneficial for ion extraction and ion detection.

Exchange of ions between ion pairs in gas phase is difficult: In solution phase, the ion pair partner of an ion can be easily displaced by another ion with the same charge in the solution, and the exchanged ions can be the same or different types of ions. For example, the ammonium ion that is paired with a phosphate ion in nucleic acid can be easily displaced by sodium and vice versa. The chloride ion that is paired with a guanidium ion in peptide can be easily displaced by a hydrogen sulfate ion and vice versa. However, in gas phase, such exchange of ions between ion pairs is expected to have a high kinetic barrier because one of the steps for the exchange is the separation of ion pairs, and the energy needed for the separation is as high as $138 \mathrm{~kJ} / \mathrm{mol}$ as analyzed earlier. Therefore, the exchange of ions between ion pairs in gas phase is difficult. This phenomenon is beneficial for ion detection as the change of metal cations of an ion pair to an ammonium cation that can transfer its proton to the counter anion has a high energy barrier.

Ion extraction: The above approach for the estimation of the ion yields of different classes of compounds is at the macroscale level based on gas phase thermal equilibrium. A different approach for the analysis is to analyze at the individual ion pair level. This 
approach may be more likely to foster insights useful for the design of experiments to improve the sensitivity and resolution of MALDI MS. At the time of ion extraction, the ion pair thermal model assumes that the plume is overall neutral, all ions are ion paired, and the relative ratios of different species have already been set by thermal equilibrium at a certain stage of the desorption and ionization process when the plum was at certain temperature and pressure. Later in the process when the pressure is lowered, new equilibrium cannot be formed. The ion pairs (as well as neutral molecules) have different energies. For a small fraction (see the following for specifics) of the ion pairs (or molecules with ion pairs), their energy is significantly higher than the average. Upon application of the ion extraction potential, the ion pairs with much higher energy are permanently separated. The separated ions go in opposite directions and become free ions. An ion may collide with another ion pair or neutral molecule before entering the field free region of the spectrometer, but this does not necessarily consume the free ion. The free ion may continue to travel to the detector, or it may pair or react with the oppositely charged ion in the ion pair and the other ion in the ion pair may reach the detector.

The typical extraction potential used in MALDI MS is in the order of $10^{6} \mathrm{~V} / \mathrm{m} .{ }^{3}$ Using 2.1 $\times 10^{6} \mathrm{~V} / \mathrm{m}$ as an example, the force exerted on a unit charge by the potential is $3.4 \times 10^{-}$ ${ }^{13} \mathrm{~N}$ (see supporting information). The attraction force between two opposite unit charges is $2.5 \times 10^{-10} \mathrm{~N}$ assuming a distance of $1 \mathrm{~nm}$ between the unit charges. Therefore, without the assistance of thermal energy of ion pairs, the ion extraction potential would not be sufficient to separate the ions in an ion pair. ${ }^{3}$ If Pauling repulsion force between the ions at the $1 \mathrm{~nm}$ distance and beyond were considered, the required force for the ion separation would be lower than $2.5 \times 10^{-10} \mathrm{~N}$. However, Pauling force decreases far more sharply with the increase of distance than the Columbic force, the repulsing force can hardly change the result. Based on these considerations, if separation of the charges solely by the ion extraction potential were desired, the distance between the two opposite unit charges would need to be $26 \mathrm{~nm}$ or longer.

The energy of an ion pair that can assist ion pair separation by ion extraction potential to generate a detectable free ion include translational kinetic energy, vibrational potentialkinetic energy and rotational kinetic energy. For translational kinetic energy to provide the assistance, collision with other molecules or ion pairs is required. Energy in the form of vibration between the two ions in the ion pair is most helpful for the assistance. Vibrational energy in other parts of the ion pair and rotational kinetic energy may provide little assistance. However, different types of internal energy can interconvert through thermal equilibrium.

Ignoring the role of Pauling repulsion force at and beyond $1 \mathrm{~nm}$, which is the assumed distance at which the Columbic attraction force and the Pauling repulsion force are balanced, the energy required to separate two opposite unit charges from $1 \mathrm{~nm}$ to $26 \mathrm{~nm}$, which is the distance required for the force from the extraction potential to overcome the attractive Coulombic force in the ion pair, is $2.22 \times 10^{-19} \mathrm{~J}$. The energy that can be provided by an ion extraction potential of $2.1 \times 10^{6} \mathrm{~V} / \mathrm{m}$ to separate two unit charges from 
$1 \mathrm{~nm}$ to $26 \mathrm{~nm}$ is $0.84 \times 10^{-19} \mathrm{~J}$. Therefore, the majority of the energy required for ion separation from an ion pair has to come from the thermal energy of the ion pair, which is $1.38 \times 10^{-19}\left(2.22 \times 10^{-19}-0.84 \times 10^{-19}\right) \mathrm{J}$.

The estimated temperature of the plum ranges from $400 \mathrm{~K}$ to $2,200 \mathrm{~K}$ or higher. ${ }^{10,52,53}$ Assuming the energy distribution among the ion pairs follows the Boltzmann equation, these temperatures correspond to a fraction of $1.4 \times 10^{-11}$ to $1.1 \times 10^{-2}$ ion pairs that have the required $1.38 \times 10^{-19} \mathrm{~J}$ thermal energy to be separable into free ions by the ion extraction potential. If the temperature of $1,000 \mathrm{~K}$ is assumed, the number is $4.6 \times 10^{-5}$. These numbers are related to the ion yields observed in MALDI MS experiments according to the ion pair thermal model.

For the case of the permanent salts such as BTPP-Cl, ${ }^{23}$ the experimentally measured ion yields, which were defined as the quotient of total number of ions detected by MS over the total number of salt molecules in the sample, are in the order of $10^{-4}$. The calculated value of $4.6 \times 10^{-5}$ at $1,000 \mathrm{~K}$ can be considered close to but slightly lower than the experimental values. One hypothesis for the discrepancy is related to the lucky survivor model, under which thermal equilibrium of plume is not fully achieved, and the distance between the ions in some of the ion pairs is longer than $1 \mathrm{~nm}$ due to shielding by other molecules, and remains so after the shielding molecules fall off. Another possibility is that the plume temperature is higher than $1,000 \mathrm{~K}$. For example, at $1,200 \mathrm{~K}$, the calculated ion yield is $2.4 \times 10^{-4}$, which matches the experimental numbers well. In addition, for more accurate analysis, the plume pressure and frequency of particle collision that is required to transform translational kinetic energy to ion separation should also be considered.

For the neutral matrix molecule CHCA, the experimental ion yield, which was defined slightly different from that of permanent salts, and is the quotient of total number of ions detected by MS over the total number of molecules including ions and neutral molecules in the sample, is in the order of $10^{-7}-10^{-8} .{ }^{23}$ These are significantly lower than the yields of permanent salts such as BTPP-Cl. The lower values are probably caused by the lower concentration of the ion pair $[\mathrm{M}-\mathrm{H}]:[\mathrm{M}+\mathrm{H}]^{+}$(see individual ion pair analysis with equation 1 in supporting information) in the plume than ion pairs of BTPP-Cl. For BTPP-Cl, $100 \%$ of the species can be assumed to exist as ion pairs in the plume. For CHCA, the majority of the molecules in the plume would be neutrals. At $1,000 \mathrm{~K}$, the ratio of ion pair over the matrix is estimated to be $10^{-13}$. The estimated ion yield would be $4.6 \times 10^{-18}\left(4.6 \times 10^{-5} \times\right.$ $\left.10^{-13}\right)$, which is significantly lower than the experimental data.

The discrepancy may be accountable by several hypotheses. One is that the plume temperature may be higher than $1,000 \mathrm{~K}$. For example, at $2,200 \mathrm{~K}$, the ratio of ion pair over the matrix becomes $10^{-6}$, and the estimated ion yield became $1.1 \times 10^{-8}\left(1.1 \times 10^{-2}\right.$ $\left.\times 10^{-6}\right)$, which is close to observed yields. The second hypothesis is that the neutralization via proton transfer process may not be able to reach thermal equilibrium, and the ion pair to neutral ratio in the plum is between $3.5 \times 10^{-2}$, the value in solution or solid phase, and $10^{-13}$, the value in gas phase at $1,000 \mathrm{~K}$. The third hypothesis is that the trace impurities such as alkaline salts in the matrix affect the ratio of ion to neutral molecules. When the 
counter cation of the carboxylate is a metal, once in the gas phase, because exchange of ions of ion pairs in gas phase is difficult, the carboxylate and metal ion pair will behave like the permanent salts. Similarly, if the counter anion of the protonated matrix is a weaker base such as hydrogen sulfate rather than the carboxylate, the ion to neutral ratio in the gas phase will also be higher. Finally, it is also possible that other ionization pathways proposed in the literature played a more important role in this case.

For peptides, the simplest case for negative peptide ion detection is that the ion pair is $\mathrm{Pep}-\mathrm{CO}_{2}^{-}: \mathrm{Na}^{+}$(or $\mathrm{K}^{+}$). If all the peptide molecules contain such an ion pair, the MALDI MS ion yield would be similar to that of permanent salts. In reality, too much salts are more of a problem for MALDI MS because it reduces the likelihood of single charged ions and increases metal adduct formation. Therefore, maintaining the majority of cations being ammonium ions is important as they can neutralize the carboxylates in the gas phase via proton transfer. For the analysis of peptide ionization using the individual ion pair approach, we can use the case of equation (5) as an example. Assuming the plume temperature is $1,000 \mathrm{~K}$, the predicted ion yield is at the order of $10^{-9}\left(4.6 \times 10^{-5} \times 10^{-4}\right)$, which is significantly lower than the experimentally determined yield of $10^{-4}-10^{-5}$. However, at 2,200 K, the predicted yield is at the order of $10^{-4}\left(1.1 \times 10^{-2} \times 3.2 \times 10^{-2}\right)$, which is consistent with the observed values. Besides temperature, in the case of large biomolecules, intramolecular stabilization of ion pair by electron donor and acceptor groups may be a reason for the higher than calculated ion yield as the stabilization can increase the ion pair to neutral ratio and making the separation of ions in ion pairs easier. Finally, like in the analysis of matrix ionization and ion extraction, it is also possible that other ionization pathways described in the literature may be at play.

For nucleic acids, the negative ions detected by MALDI MS are most likely coming from the Nuc- ${ }_{3} \mathrm{PO}: \mathrm{Na}^{+}$(or $\mathrm{K}^{+}$) ion pair rather than the $\mathrm{Nuc}_{-}-\mathrm{O}_{3} \mathrm{PO}: \mathrm{NH}_{4}{ }^{+}$ion pair, as the concentration of the latter in gas phase is too low for detection due to the strong tendency for proton transfer. For the ion yield of $\mathrm{Nuc}_{-} \mathrm{O}_{3} \mathrm{PO}: \mathrm{Na}^{+}$, the analysis for permanent salts can be applied. The predicted ion yield would be at the order of $10^{-4}\left(4.6 \times 10^{-4} \times 10\right)$ assuming each nucleic acid molecule contains over 10 nucleotides, the temperature is $1,000 \mathrm{~K}$ and there are sufficient alkaline metal cations (but not too much to cause multicharged or metal adduct peaks) needed for the formation of the $\mathrm{Nuc}^{-} \mathrm{O}_{3} \mathrm{PO}^{-} \mathrm{Na}^{+}$(or $\mathrm{K}^{+}$) ion pair. For the case of positive ion detection, the ion yield analysis is similar to that of positive peptide ion detection.

For neutral molecules as described in equation (13), at $1,000 \mathrm{~K}$, the gas phase ratio of ion pair $\mathrm{AH}^{+}: \mathrm{X}$ - over neutral $\mathrm{A}$ is $3.2 \times 10^{-8}$, and predicted ion yield is $10^{-14}\left(4.6 \times 10^{-5} \times\right.$ $\left.3.2 \times 10^{-8}\right)$. At $2,200 \mathrm{~K}$, the gas phase ratio of ion pair $\mathrm{AH}^{+}: \mathrm{X}^{-}$over neutral $\mathrm{A}$ is $10^{-4}$, and the predicted ion yield is $10^{-6}\left(1.1 \times 10^{-2} \times 10^{-4}\right)$, which is in the range detectable by MS detector.

It is noted that the above calculations and analyses cannot be accurate. The ions are treated as point charges, the Pauling repulsion force is not considered, the distribution of energy in different degrees of freedom of motion is ignored, the interaction of species 
shown in an equation with those not in the equation is not considered, and the ion pairs are assumed completely stable during desorption and ionization. All these can cause inaccuracy. In addition, the distance between the ions at which the attraction and repulsion forces cancel each other may be significantly different from $1 \mathrm{~nm}$ and vary widely with different ion pairs, and the temperature of the plume is difficult to determine, and these two parameters affect the results of ion yield calculation exponentially. The intend of the calculation and more importantly the analysis is to give a rough picture of the ion extraction process in MALDI MS. Such a picture can be helpful for designing systems to improve MALDI MS sensitivity and resolution.

The above discussion is focused on the situation of an overall neutral plume. For cases that the plume is not overall charge neutral, upon application of the ion extraction potential, the free ions that constitute the excess charges will either be easily extracted by the potential and detected by the MS detector or be driven to the undesired direction. In both cases, the plume will then become neutral, and the same situation as described for a neutral plume will follow.

Detection of predominantly single-charged ions: The ion pair thermal model can help to explain several important observations in MALDI MS. The detection of predominantly single-charged ions, which is interesting and a significant advantage of MALDI MS over ESI MS, is one of them. Even for molecules that have multiple easily ionizable groups such as nucleic acids and peptides, single-charged ions almost always predominate. Double- and triple-charged ions may be detected but their peaks are almost always less intense. In the context of the ion pair thermal model, in the plume, there is a strong tendency for the molecules to exist in a neutral form, either through proton transfer or through ion pair. Taking equation (5) for an example, at 1,000 K, for a guanidine group to be protonated and detected, the chance is $10^{-4}$ for Pep- $\mathrm{NH}^{+}: \mathrm{HSO}_{3}{ }^{-}$over Pep-N. At 2,200 $\mathrm{K}$, the number is $3.2 \times 10^{-2}$. For a molecule that contains two such ion pairs, the chance for the molecule to appear as a double-charged peak in MALDI MS is $2.1 \times 10^{-19}\left(10^{-4} \times\right.$ $\left.10^{-4} \times 4.6 \times 10^{-5} \times 4.6 \times 10^{-5}\right)$ at $1,000 \mathrm{~K}$, and $1.2 \times 10^{-9}\left(3.2 \times 10^{-2} \times 3.2 \times 10^{-2} \times 1.1 \times\right.$ $10^{-2} \times 1.1 \times 10^{-2}$ ) at $2,200 \mathrm{~K}$. In reality, the chance for detecting doubled charged ions is significantly higher, which indicates that thermal equilibrium may not be fully reached in the plume, and some pre-existed ions in the sample, which have larger inter-ion distance in ion pair after they ejected into the plume, may contribute to the MS signal.

The total number of ions detected is roughly constant: The ion pair neutralization model can provide an explanation for the observation that the total numbers of ions detected by the MS detector are roughly constant irrespective of the molar ratio of an analyte over matrix in a MALDI MS sample and the identity of the analyte. ${ }^{23,34}$ Taking the analysis of BTPP-Cl with MALDI MS as an example, when the sample composition was $0.1 \mathrm{pmol}$ analyte in $25 \mathrm{nmol} \mathrm{CHCA}$, the ion counts of $\mathrm{BTPP}^{+}$and $[\mathrm{CHCA}+\mathrm{H}]^{+}$were 0.52 $\times 10^{8}$ and $1.8 \times 10^{9}$, respectively; when the analyte was increased to $3 \mathrm{pmol}$, the ion counts were $11 \times 10^{8}$ and $1.4 \times 10^{9}$, respectively; and when analyte was $30 \mathrm{pmol}$, the ion counts were $28 \times 10^{8}$ and $0.29 \times 10^{9}$, respectively. ${ }^{23}$ These data indicate that the total 
number of ions including those from the analyte and matrix do not change significantly when the ratio of analyte over matrix changes significantly. In addition, it was observed that the total number of ions does not exceed the total number of matrix ions obtained with pure matrix. In certain ranges of analyte concentration in the sample, as the concentration increases, the number of analyte ions increases, but it is at the cost of the number of matrix ions, which decreases. Similar experimental observations were reported for other ionic compounds and peptides. ${ }^{34}$ In the context of the ion pair thermal model, three factors may be accountable for the observations. One is that the fraction of molecules including ion pairs and neutral molecules of analyte and matrix that have sufficient thermal energy to render free ion generation is set by the plume temperature. At certain range, the analyte over matrix ratio has little influence on the fraction number. The other is that when the concentration of ion pairs that have sufficient energy to be separable by the ion extraction potential to become free ions dispersed in the plume of mainly neutral matrix molecules exceeds certain limit, they are more likely to collide with each other and become neutral molecules via proton transfer.

Detection of positive and negative ions under the same desorption and ionization conditions: For nucleic acid and peptide analytes as well as other molecules, which carry both positively and negatively charged groups, detection of positive and negative ions under the same desorption and ionization conditions is usually possible. ${ }^{26}$ In some reports, similar yields/peak intensities for positive and negative ions were observed. Models have been proposed to explain the observations. For example, the energy transfer induced disproportionation assumes that in the solid sample, two analyte molecules share an active proton through hydrogen bonding. A closely located excited matrix molecule transfers its energy to the analyte dimer to induce disproportionation, which generates one protonated analyte molecule and one deprotonated analyte ion. ${ }^{26}$ In the context of the ion pair thermal model, where free ions in the plume are not considered a requirement for ion detection, and ion pairs with sufficient energy are mainly responsible for ion detection, these observations can be easily explained. In the plume, both types of ion pairs with analyte being positive ions and those with analytes being negative ions exist, and in some cases with close to equal quantity. Their energies are about the same, and thus the chance for their separation by ion extraction potential to become detectable free ions is about the same. Therefore, the detection of positive and negative analyte ions under the same desorption and ionization is observed.

Reduction of metal adducts in nucleic acid analysis using ammonium salt as comatrices: In MALDI MS analysis of nucleic acids, desalting is usually critical for success. This is especially true for the analysis of nucleic acids longer than 60 nucleotides. In many cases, various adducts of multiple metal cations such as potassium and sodium can become so prevalent that the analysis can provide little information. The abundance of the adducts may also prevent desorption. ${ }^{54-56}$ Ammonium salts such as diammonium hydrogen citrate have been found to be able to alleviate these problems when they are used as co-matrices, although long nucleic acid analysis is still challenging. In the literature, the formation of complex metal adducts in nucleic acid analysis is usually 
attributed to the high affinity of the alkaline metal cations toward the phosphate groups of nucleic acids. In the context of the ion pair thermal model, the metal adducts can be conceived to be easy to form because of the strong Coulombic attraction force between the metal cations and the phosphates in the plume. Few of the multiple ion pairs in a nucleic acid molecule have sufficient thermal energy to become separable by the ion extraction potential. Ammonium salt co-matrices can alleviate the problem because with ammonium salt in the solid sample, the chance for the phosphate groups to ion pair with alkaline metal cations is lowered, and most or almost all the phosphate groups could pair with ammonium cations. Once in the gas phase, the ammonium and alkaline metal cations in the ion pairs are not easy to exchange, and the ion pairs with ammonium cation can readily neutralize via proton transfer.

Ion suppression: In MALDI MS, occasionally when the analyte concentration in a sample reaches certain levels, matrix ions can be partially or even completely suppressed. In addition, suppression of one analyte ion by another analyte ion formed from the same or a different analyte can also occur. Because MALDI MS samples are heterogeneous, and samples of an analyte prepared using the same recipe can give variable analysis results, special experimental design such as closely matched controls, experiments with standard samples and statistically meaningful amount of data are needed if MALDI MS is to be used for analyte quantification and for the determination of analyte purity. ${ }^{7,9,26,57}$ In the context of ion pair thermal model, ion suppression is conceivable. The total number of ion pairs that have sufficient energy to generate detectable free ions by the extraction potential is roughly constant. Taking the suppression of matrix ions by analyte ions for an example, when the concentration of an analyte in the sample increases, the number of high energy ion pairs involving an ion from the analyte in the plume increases. This increase is at the cost of the high energy ion pairs involving matrix ions, which become undetectable species via mechanisms such as neutralization via proton transfer and loss of kinetic and vibrational energy.

\section{Conclusions}

In summary, the ion pair thermal model for MALDI MS is proposed. Key assumptions of the model include high temperature plume, gas phase thermal equilibrium, strong tendency to gas phase neutralization via ion pair formation and proton transfer, and overall plume charge neutral. The quantities of ions in the solid sample and in the gaseous plume were estimated. Ion yields were estimated based on gas phase thermal equilibrium at the macroscale level and at the individual ion pair level. The estimated ion yields were close to but might be somewhat lower than experimentally determined values depending on the assumption of plume temperature. One possible explanation for the lower than observed ion yields can be deviation of full thermal equilibrium of the actual plume. Besides ion yield estimation, the model can provide explanation of some observations in MALDI MS, which include detection of single-charged ions, roughly constant total number of ions, equal detection of positive and negative ions, reduction of metal adducts with ammonium salt and ion suppression. We hope that the new model can provide guidance 
on the design of new conditions and systems that can improve the performance of MALDI MS such as improving its sensitivity and resolution, and its ability and reliability for the analysis of large biomolecules.

\section{Author Information}

Corresponding author: Shiyue Fang, Department of Chemistry, Michigan Technological University, 1400 Townsend Drive, Houghton, MI 49931 USA. Tel: 906-487-2023. Email: shifang@mtu.edu

\section{Notes}

The author declares no competing financial interest.

\section{Acknowledgement}

Financial support from NSF (CHE1954041) and NIH (GM109288) is gratefully acknowledged.

\section{Supporting Information}

All calculations to derive the quantities of ions and ion pairs in solid sample and in gas phase, gas phase equilibrium constants, and MALDI MS ion yields of different classes of molecules. 


\section{References}

1. Moon, J. H.; Yoon, S.; Bae, Y. J.; Kim, M. S., Formation of Gas-Phase Peptide lons and Their Dissociation in Maldi: Insights from Kinetic and Ion Yield Studies. Mass Spectrometry Reviews 2015, 34 (2), 94-115.

2. Bae, Y. J.; Kim, M. S., A Thermal Mechanism of Ion Formation in MALDI. Annu Rev Anal Chem (Palo Alto Calif) 2015, 8, 41-60.

3. Zenobi, R.; Knochenmuss, R., Ion formation in MALDI mass spectrometry. Mass spectrometry reviews $1998,17(5), 337-366$.

4. Karas, M.; Kruger, R., Ion formation in MALDI: the cluster ionization mechanism. Chem Rev 2003, 103 (2), 427-40.

5. Lai, Y. H.; Wang, Y. S., Matrix-Assisted Laser Desorption/Ionization Mass Spectrometry: Mechanistic Studies and Methods for Improving the Structural Identification of Carbohydrates. Mass Spectrom (Tokyo) 2017, 6 (Spec Iss 2), S0072.

6. Oberacher, $\mathrm{H}$., On the use of different mass spectrometric techniques for characterization of sequence variability in genomic DNA. Anal Bioanal Chem 2008, 391 (1), 135-149.

7. Dreisewerd, K., Recent methodological advances in MALDI mass spectrometry. Anal Bioanal Chem 2014, 406 (9-10), 2261-2278.

8. Knochenmuss, R., The Coupled Chemical and Physical Dynamics Model of MALDI. Annu Rev Anal Chem 2016, 9, 365-385.

9. Knochenmuss, R., lon formation mechanisms in UV-MALDI. Analyst 2006, 131 (9), 966-986.

10. Yoon, S. H.; Moon, J. H.; Kim, M. S., A Comparative Study of In- and Post-Source Decays of Peptide and Preformed lons in Matrix-Assisted Laser Desorption Ionization Time-of-Flight Mass Spectrometry: Effective Temperature and Matrix Effect. J Am Soc Mass Spectr 2010, 21 (11), 1876-1883.

11. Kinsel, G. R.; Yao, D. Q.; Yassin, F. H.; Marynick, D. S., Equilibrium conditions in laser-desorbed plumes: thermodynamic properties of alpha-cyano-4-hydroxycinnamic acid and protonation of amino acids. Eur J Mass Spectrom 2006, 12 (6), 359-367.

12. Karas, M.; Gluckmann, M.; Schafer, J., Ionization in matrix-assisted laser desorption/ionization: singly charged molecular ions are the lucky survivors. J Mass Spectrom 2000, 35 (1), 1-12.

13. Jaskolla, T. W.; Karas, M., Compelling evidence for Lucky Survivor and gas phase protonation: the unified MALDI analyte protonation mechanism. J Am Soc Mass Spectrom 2011, 22 (6), 976-88.

14. Land, C. M.; Kinsel, G. R., Investigation of the mechanism of intracluster proton transfer from sinapinic acid to biomolecular analytes. J Am Soc Mass Spectr 1998, 9 (10), 1060-1067.

15. Knochenmuss, R., A quantitative model of ultraviolet matrix-assisted laser desorption/ionization. Journal of Mass Spectrometry 2002, 37 (8), 867-877.

16. Lin, H. Y.; Song, B.; Lu, I. C.; Hsu, K. T.; Liao, C. Y.; Lee, Y. Y.; Tseng, C. M.; Lee, Y. T.; Ni, C. K., Is energy pooling necessary in ultraviolet matrix-assisted laser desorption/ionization? Rapid Communications in Mass Spectrometry 2014, 28 (1), 77-82.

17. Breuker, K.; Knochenmuss, R.; Zenobi, R., Gas-phase basicities of deprotonated matrix-assisted laser desorption/ionization matrix molecules. Int J Mass Spectrom 1999, 184 (1), 25-38.

18. Chen, X. J.; Carroll, J. A.; Beavis, R. C., Near-ultraviolet-induced matrix-assisted laser desorption/ionization as a function of wavelength. J Am Soc Mass Spectr 1998, 9 (9), 885-891.

19. Niu, S. F.; Zhang, W. Z.; Chait, B. T., Direct comparison of infrared and ultraviolet wavelength matrix-assisted laser desorption/ionization mass spectrometry of proteins. J Am Soc Mass Spectr 1998, 9 (1), 1-7.

20. Zhu, Y.; Lee, K.; Tang, K.; Allman, S.; Taranenko, N.; Chen, C., Revisit of MALDI for small proteins. Rapid communications in mass spectrometry 1995, 9 (13), 1315-1320. 
21. Kruger, R.; Pfenninger, A.; Fournier, I.; Gluckmann, M.; Karas, M., Analyte incorporation and ionization in matrix-assisted laser desorption/ionization visualized by $\mathrm{pH}$ indicator molecular probes. Analytical Chemistry 2001, 73 (24), 5812-5821.

22. Liao, P. C.; Allison, J., Enhanced detection of peptides in matrix-assisted laser desorption/ionization mass spectrometry through the use of charge-localized derivatives. Journal of Mass Spectrometry 1995, 30, 511-512.

23. Moon, J. H.; Shin, Y. S.; Bae, Y. J.; Kim, M. S., lon Yields for Some Salts in MALDI: Mechanism for the Gas-Phase Ion Formation from Preformed lons. J Am Soc Mass Spectr 2012, 23 (1), 162-170.

24. Krüger, R.; Pfenninger, A.; Fournier, I.; Glückmann, M.; Karas, M., Analyte incorporation and ionization in matrix-assisted laser desorption/ionization visualized by $\mathrm{pH}$ indicator molecular probes. Analytical chemistry 2001, 73 (24), 5812-5821.

25. Alonso, E.; Zenobi, R., Non-linear photoelectron effect contributes to the formation of negative matrix ions in UV-MALDI. Phys Chem Chem Phys 2016, 18 (29), 19574-87.

26. Chang, W. C.; Huang, L. C.; Wang, Y. S.; Peng, W. P.; Chang, H. C.; Hsu, N. Y.; Yang, W. B.; Chen, C. H., Matrix-assisted laser desorption/ionization (MALDI) mechanism revisited. Anal Chim Acta 2007, 582 (1), 1-9.

27. Yang, C.; Hu, X.; Loboda, A. V.; Lipson, R. H., A useful binary matrix for visible-MALDI of low molecular weight analytes. J Am Soc Mass Spectrom 2010, 21 (2), 294-9.

28. J.-D. Yang, J.-P. C., Internet Bond-Energy Databank (PKa and BDE) -IBonD, v2.0.

29. Kütt, A.; Selberg, S.; Kaljurand, I.; Tshepelevitsh, S.; Heering, A.; Darnell, A.; Kaupmees, K.; Piirsalu, M.; Leito, I., pKa values in organic chemistry-Making maximum use of the available data. Tetrahedron Letters 2018, 59 (42), 3738-3748.

30. Most thermodynamic data in this article are taken from NIST Chemistry WebBook, NIST Standard Reference Database; National Institute of Standards and Technology: http://webbook.nist.gov. http://webbook.nist.gov.

31. Caldwell, G.; Renneboog, R.; Kebarle, P., Gas phase acidities of aliphatic carboxylic acids, based on measurements of proton transfer equilibria. Canadian Journal of Chemistry 1989, 67 (4), 611-618.

32. Lias, S. G.; Liebman, J. F.; Levin, R. D., Evaluated gas phase basicities and proton affinities of molecules; heats of formation of protonated molecules. J Phys Chem Ref Data 1984, 13 (3), 695-808.

33. Hunter, E. P. L.; Lias, S. G., Evaluated gas phase basicities and proton affinities of molecules: An update. J Phys Chem Ref Data 1998, 27 (3), 413-656.

34. Bae, Y. J.; Shin, Y. S.; Moon, J. H.; Kim, M. S., Degree of Ionization in MALDI of Peptides: Thermal Explanation for the Gas-Phase Ion Formation. J Am Soc Mass Spectr 2012, 23 (8), 1326-1335.

35. Wang, X. B.; Nicholas, J. B.; Wang, L. S., Photoelectron spectroscopy and theoretical calculations of SO4- and HSO4-: Confirmation of high electron affinities of SO4 and HSO4. J Phys Chem A 2000, 104 (3), 504-508.

36. Morris, R. A.; Knighton, W. B.; Viggiano, A. A.; Hoffman, B. C.; Schaefer, H. F., The gas-phase acidity of H3PO4. J Chem Phys 1997, 106 (9), 3545-3547.

37. Moser, A.; Range, K.; York, D. M., Accurate Proton Affinity and Gas-Phase Basicity Values for Molecules Important in Biocatalysis. J Phys Chem B 2010, 114 (43), 13911-13921.

38. Lum, R. C.; Grabowski, J. J., Trimethyl Phosphate - the Intrinsic Reactivity of Carbon Versus Phosphorus Sites with Anionic Nucleophiles. Journal of the American Chemical Society 1992, 114 (22), 8619-8627.

39. Aue, D. H.; Webb, H. M.; Bowers, M. T., A thermodynamic analysis of solvation effects on the basicities of alkylamines. An electrostatic analysis of substituent effects. Journal of the American Chemical Society 1976, $98(2), 318-329$.

40. Bouchoux, G.; Huang, S. H.; Inda, B. S., Acid-base thermochemistry of gaseous aliphatic alphaaminoacids. Physical Chemistry Chemical Physics 2011, 13 (2), 651-668. 
41. https://doi.org/10.1021/jp8068877.

42. Moser, A.; Range, K.; York, D. M., Accurate proton affinity and gas-phase basicity values for molecules important in biocatalysis. J Phys Chem B 2010, 114 (43), 13911-21.

43. Arnett, E. M.; Quirk, R. P.; Burke, J. J., Weak bases in strong acids. III. Heats of ionization of amines in fluorosulfuric and sulfuric acids. New general basicity scale. journal of the American Chemical Society 1970, 92 (5), 1260-1266.

44. Deno, N. C.; Turner, J. O., The Basicity of Alcohols and Ethers. Journal of Organic Chemistry 1966, 31 (6), 1969-1970.

45. Jozwiak, M.; Piekarski, H.; Jozwiak, A., Thermodynamics of interactions of Na+ ion with 15-crown5 ether in the mixtures of water with dimethylsulfoxide at $298.15 \mathrm{~K}$. J Mol Liq 2003, 106 (1), 15-29.

46. Jozwiak, M., Complex formation of crown ethers with cations in the water-organic solvent mixtures. Part VIII. Thermodynamic of interactions of $\mathrm{Na}+$ ion with 15 -crown-5 and benzo-15-crown-5 ethers in the mixtures of water with hexamethylphosphortriamide at $298.15 \mathrm{~K}$. J Solution Chem 2004, 33 (9), 1073-1084.

47. Izatt, R. M.; Terry, R. E.; Haymore, B. L.; Hansen, L. D.; Dalley, N. K.; Avondet, A. G.; Christensen, J. J., Calorimetric titration study of the interaction of several uni- and bivalent cations with 15-crown-5, 18-crown-6, and two isomers of dicyclohexo-18-crown- 6 in aqueous solution at 25 .degree.C and $\cdot \mathrm{mu}$. $=$ 0.1. Journal of the American Chemical Society 1976, 98 (24), 7620-7626.

48. Lias, S. G.; Liebman, J. F.; Levin, R. D., Evaluated Gas-Phase Basicities and Proton Affinities of Molecules - Heats of Formation of Protonated Molecules. J Phys Chem Ref Data 1984, 13 (3), 695-808.

49. Evans, J.; Nicol, G.; Munson, B., Proton affinities of saturated aliphatic methyl esters. J Am Soc Mass Spectr 2000, 11 (9), 789-796.

50. Blake, J. F.; Jorgensen, W. L., Proton Affinities and Gas-Phase Basicities of Alkyl and Silyl Ethers. Journal of Organic Chemistry 1991, 56 (21), 6052-6059.

51. Armentrout, P. B.; Austin, C. A.; Rodgers, M. T., Alkali Metal Cation Interactions with 15-Crown-5 in the Gas Phase: Revisited. J Phys Chem A 2014, 118 (37), 8088-8097.

52. Spengler, B., Post-source decay analysis in matrix-assisted laser desorption/ionization mass spectrometry of biomolecules. Journal of Mass Spectrometry 1997, 32 (10), 1019-1036.

53. Bae, Y. J.; Moon, J. H.; Kim, M. S., Expansion Cooling in the Matrix Plume is Under-Recognized in MALDI Mass Spectrometry. J Am Soc Mass Spectr 2011, 22 (6), 1070-1078.

54. Koomen, J. M.; Russell, W. K.; Hettick, J. M.; Russell, D. H., Improvement of Resolution, Mass Accuracy, and Reproducibility in Reflected Mode DE-MALDI-TOF Analysis of DNA Using Fast EvaporationOverlayer Sample Preparations. Analytical chemistry 2000, 72 (16), 3860-3866.

55. Sauer, S., The essence of DNA sample preparation for MALDI mass spectrometry. Journal of biochemical and biophysical methods 2007, 70 (2), 311-318.

56. Smirnov, I. P.; Hall, L. R.; Ross, P. L.; Haff, L. A., Application of DNA-binding polymers for preparation of DNA for analysis by matrix-assisted laser desorption/ionization mass spectrometry. Rapid Communications in Mass Spectrometry 2001, 15 (16), 1427-1432.

57. Kong, Y.; Zhu, Y.; Zhang, J. Y., lonization mechanism of oligonucleotides in matrix-assisted laser desorption/ionization time-of-flight mass spectrometry. Rapid Communications in Mass Spectrometry 2001, 15 (1), 57-64. 